\title{
Forward Physics at the LHC: within and beyond the Standard Model
}

\author{
David d'Enterria \\ CERN, PH, CH-1211 Geneva 23, Switzerland \\ E-mail:dde@cern.ch
}

(Received on 4 June, 2008)

\begin{abstract}
We review the detection capabilities in the forward direction of the various LHC experiments together with the associated physics programme. A selection of measurements accessible with near-beam instrumentation in various sectors (and extensions) of the Standard Model (SM) is outlined, including QCD (diffractive and elastic scattering, low- $x$ parton dynamics, hadronic Monte Carlos for cosmic-rays), electroweak processes in $\gamma \gamma$ interactions, and Higgs physics (vector-boson-fusion and central exclusive production).
\end{abstract}

Keywords: LHC; Forward detectors; $p$ - $p$; $P b-P b$; QCD; Electroweak sector; Exclusive Higgs boson

\section{INTRODUCTION}

The CERN Large Hadron Collider (LHC) will deliver proton-proton, proton-nucleus and nucleus-nucleus collisions at $\sqrt{{S_{N N}}_{1}}=5.5,8.8$ and $14 \mathrm{TeV}$ respectively, opening up an unprecedented phase-space for particle production spanning up to $\Delta \eta \sim 20$ units of rapidity. Many interesting scattering processes (mostly mediated by colorless exchanges) are characterized by particles emitted at very low angles with respect to the beam. Figure 1 shows a few representative diagrams mediated respectively by (a) partons, (b) a photon and a Pomeron, (c) two photons, and (d) two gluons in a colorsinglet state. All these processes are characterized by forward particles (jets, protons, ions) in the final-state plus an (often exclusive) system produced at more central rapidities. We present a summary of the physics programme accessible with the forward instrumentation capabilities available in the six LHC experiments: ALICE, ATLAS, CMS, LHCb, LHCf and TOTEM.

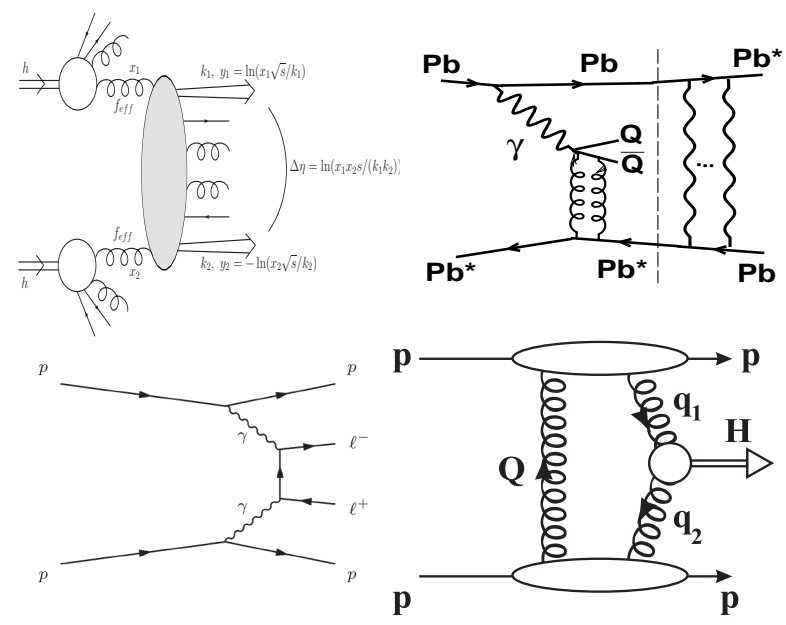

FIG. 1: Diagrams depicting processes in $\mathrm{p}-\mathrm{p}$ or $\mathrm{Pb}-\mathrm{Pb}$ collisions at the LHC characterized by forward particle emission: (a) MuellerNavelet dijets, (b) exclusive quarkonia photoproduction, (c) exclusive dileptons, (d) central exclusive Higgs.
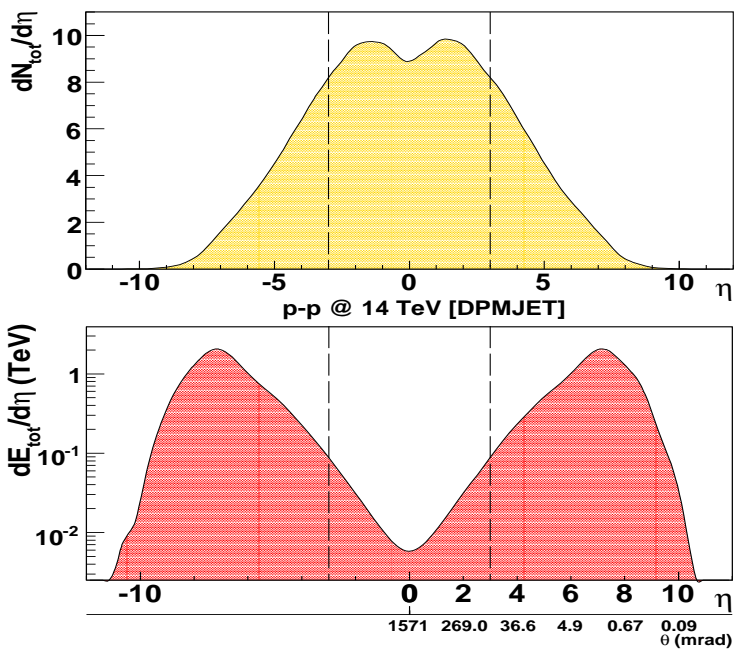

FIG. 2: Pseudo-rapidity distributions for the total hadron multiplicity (top) and energy (bottom) in p-p at $\sqrt{s}=14 \mathrm{TeV}$ as given by the DPMJET3 model [1].

\subsection{Forward kinematics variables}

The appropriate kinematic variables in (inelastic) hadronic collisions are the transverse momentum, $p_{T}=p \sin \theta$, and the rapidity $y=0.5 \log \left(E+p_{L} / E-p_{L}\right)$ or $y=\operatorname{atanh}\left(p_{L} / E\right)$, where $p_{L}=p \cos \theta$ is the longitudinal momentum, and $\theta$ is the polar angle with respect to the beam axis. The rapidity can be thought of as the relativistically-invariant measure of the longitudinal velocity. Often the pseudorapidity $\eta=-\ln \tan (\theta / 2)$ or $\eta=\operatorname{atanh}\left(p_{L} / p\right)$, which depends solely on $\theta$, is used (note that $y \approx \eta$ for negligible masses i.e. for $E \approx p$, provided that $\theta$ is not very small). From Fig. 2, one can see that particle production in hadronic collisions is peaked at $\eta=0$ (i.e. at $\left.90^{\circ}\right)^{1}$, while most of the energy is carried out by particles not far from the beam rapidity, $y_{\max }=\ln \left(\sqrt{s} / m_{p}\right)=9.54$ at $14 \mathrm{TeV}$. In elastic or diffractive collisions, one deals with particles scattered at very small angles and $\eta$ is less useful a variable (ultimately, $\eta \rightarrow \infty$ for $\theta \approx 0$ ). Instead, the Feynman $x_{F}=2 p_{L} / \sqrt{s}$

\footnotetext{
${ }^{1}$ Note that $d N / d y$ (not shown) has indeed a Gaussian shape with maximum at $y=0$. The dip in $d N / d \eta$ is just an "artifact" due to the transformation from rapidity to pseudorapidity $(d y / d \eta=p / E$ Jacobian $)$.
} 
or equivalently $\xi \approx 1-x_{F}$, and the four-momentum transfer $-t \approx(p \theta)^{2} \approx p_{T}^{2}$, are used.

\subsection{Forward detectors at the LHC}

If one (somewhat arbitrarily) defines "forward" rapidities as those beyond $|\eta| \approx 3$, all LHC experiments feature forward detection capabilities without parallel compared to previous colliders (Figs. 3 and 4):

- ATLAS [3] and CMS [4, 5] not only cover the largest $p_{T}-\eta$ ranges at mid-rapidity for hadrons, electrons, photons and muons, but they feature extended instrumentation at distances far away from the interaction point (IP). Forward calorimetry is available at $\pm 11 \mathrm{~m}$ (the FCal and HF [6] hadronic calorimeters), at $\pm 14 \mathrm{~m}$ (CMS CASTOR sampling calorimeter) [7], and at $\pm 140 \mathrm{~m}$ (the Zero-Degree-Calorimeters, ZDCs) [8, 9]. In addition, ATLAS has (or will have) Roman Pots (RPs) at $\pm 220,240 \mathrm{~m}[10,11]$, and there are advanced plans to install a new proton-tagger system at $420 \mathrm{~m}$ (FP420) from both the ATLAS and CMS IPs [12].

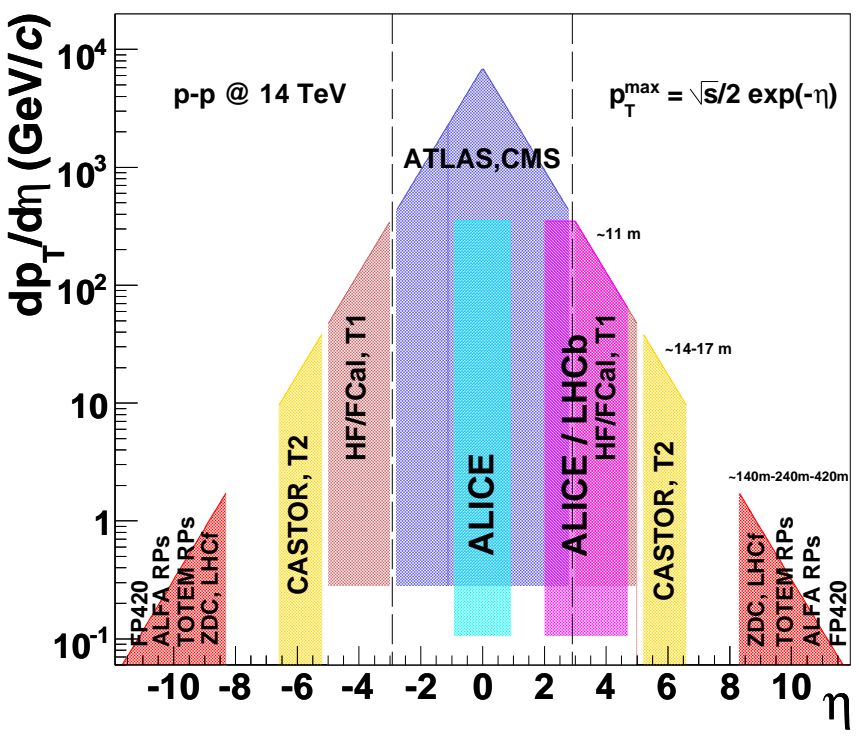

FIG. 3: Approximate $p_{T}-\eta$ coverage of current (and proposed) detectors at the LHC (adapted from [2]).

- Both ALICE [13] and LHCb [14] have forward muon spectrometers in regions, $2 \lesssim \eta \lesssim 5$, not covered by ATLAS or CMS. In addition, LHCb has good tracking, calorimetry and particle identification for the measurement of hadrons, electrons and photons in this $\eta$ range; and ALICE has also ZDCs at $\pm 116 \mathrm{~m}$ [15].

- The TOTEM experiment [16], sharing IP5 with CMS, features two types of trackers (T1 and T2 telescopes) covering $3.1<|\eta|<4.7$ and $5.2<|\eta|<6.5$ respectively, plus proton-taggers (Roman Pots) at \pm 147 and $\pm 220 \mathrm{~m}$
- The LHCf experiment [17] has installed scintillator/silicon calorimeters in the same region of the ATLAS ZDCs, $\pm 140 \mathrm{~m}$ away from IP1.

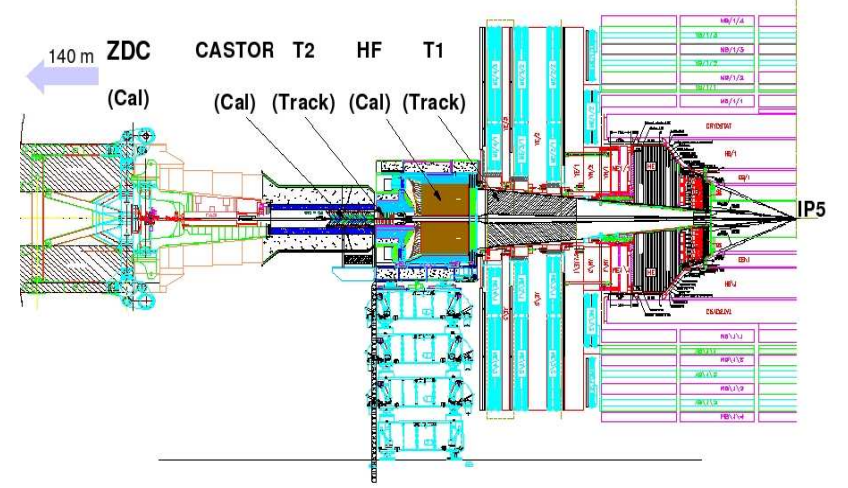

FIG. 4: Layout of the detectors in the CMS/TOTEM forward region at the LHC interaction point 5 [5].

A rich variety of physics measurements are accessible utilizing such forward instrumentation in three possible detection modes:

1. as detectors to directly measure the 4-momentum of a given final-state produced in the reaction e.g. a jet in CASTOR, a zero-degree photon in LHCf/ZDC, or a leading proton in FP420;

2. as tagging devices to signal the presence of a diffractively or elastically scattered proton (or a neutron) in Roman Pots (or ZDCs);

3. as vetoing devices of final-state particles in the collision, e.g. requiring no hadronic activity within a given (forward) rapidity range covered by one or more detectors.

\subsection{Forward physics at the LHC}

The following QCD, electro-weak, and Higgs physics topics, developed in more detail in the remainder of the document, can be studied with forward instrumentation:

a. QCD physics (Section 2). Many aspects of the physics of the strong interaction can be studied with forward detectors. First, Pomeron-induced processes [18, 19] - such as elastic $p-p$ cross section, soft diffractive processes, rapidity-gap survival probability, hard diffraction cross sections, etc. - are accessible with the TOTEM and ATLAS Roman Pots and/or by requiring a large enough rapidity gap in one (or both) of the forward hemispheres (e.g. HF+CASTOR in CMS). Second, low- $x$ QCD physics $[20,21]$ - gluon saturation, non-linear QCD evolution, small- $x$ parton distribution functions (PDFs), multi-parton scattering - can be studied via the measurement of hard QCD cross sections in the forward direction (e.g. jets, or direct- $\gamma$ in HF/FCal, CASTOR) or in 
exclusive photoproduction $\gamma$-p [22] and $\gamma$-A [23] processes, tagged with forward protons (neutrons) in RPs (ZDCs). Third, hadronic models of ultra-high-energy (UHE) cosmic-rays interactions in the upper atmosphere can be effectively tuned by measuring the forward energy $(d E / d \eta)$ and particle $(d N / d \eta)$ flows in $p-p, p-A$, and $A-A$ collisions [24].

$b$. Electroweak sector (Section 3). All charges accelerated at very high energies generate electromagnetic fields which, in the equivalent photon approximation, can be considered as (quasi-real) photon beams. A significant fraction of the $p-p$ [22] and $P b-P b$ [23] collisions at the LHC will thus involve photon interactions at $\mathrm{TeV}$ energies giving access to a unique programme of $\gamma$-induced studies. In particular, photon-photon interactions, tagged with forward protons (neutrons) in the RPs (ZDCs), allow one to measure the beam luminosity via the pure QED process $\gamma \gamma \rightarrow l^{+} l^{-}$; or to study (anomalous) gauge boson couplings via $\gamma-\mathrm{p}, \gamma-\mathrm{A} \rightarrow p n W$, or $\gamma \gamma \rightarrow Z Z, W W$.

c. Higgs physics (Section 4). Two mechanisms of Higgs production at the LHC are accompanied by forward particle emission. First, the vector-boson-fusion (VBF) process, $p p \rightarrow q q \rightarrow q H q$, where the two valence quarks radiate $W$ or $Z$ bosons which merge to form the Higgs, and then fragment into two forward-backward jets tagged in the forward calorimeter systems. Second, the central exclusive channel, $p p \rightarrow p H p$ (diagram (d) of Fig. 1), where the Higgs boson is produced at central rapidities from the fusion of a two-gluon (color-singlet) system [25] and the interacting protons, scattered intact at very small angles, are measured e.g. in the planned FP420 proton spectrometer [12].

\section{QCD PHYSICS}

\subsection{Elastic scattering}

The measurement at the LHC of the total $p-p$ cross section and of the $\rho$-parameter (the ratio of real to imaginary part of the forward elastic scattering amplitude) provides a valuable test of fundamental quantum mechanics relations such as the Froissart bound $\sigma_{t o t}<$ Const $\ln ^{2} s$, the optical theorem $\sigma_{t o t} \sim \operatorname{Im} f_{e l}(t=0)$, and dispersion relations $\operatorname{Re} f_{e l}(t=0) \sim \operatorname{Im} f_{e l}(t=0)$ [26]. The current extrapolations of the total $p$ - $p$ cross section at the LHC $\left(\sigma_{t o t}=90-\right.$ $140 \mathrm{mb}$ ), of which the elastic contribution accounts for about one fourth, suffer from large uncertainties due to a $2.6 \sigma$ disagreement between the E710 and CDF measurements at Tevatron (Fig. 5, top).

The main goal of TOTEM experiment is to obtain a precise measurement of the total and elastic $p$ - $p$ cross sections over a large range of 4-momentum transfers from $-t \approx 2 \cdot 10^{-3} \mathrm{GeV}^{2}$ to $8 \mathrm{GeV}^{2}$, using different $\beta^{*}$ optics settings (Fig. 5, bottom). The total $p$ - $p$ cross section and the LHC luminosity will be measured making use of the optical theorem via

$$
\sigma_{t o t}=\frac{16 \pi}{1+\rho^{2}} \cdot \frac{d N_{e l} /\left.d t\right|_{t=0}}{N_{e l}+N_{\text {inel }}}, \quad \mathcal{L}=\frac{1+\rho^{2}}{16 \pi} \cdot \frac{\left(N_{e l}+N_{\text {inel }}\right)^{2}}{d N_{e l} /\left.d t\right|_{t=0}} .
$$
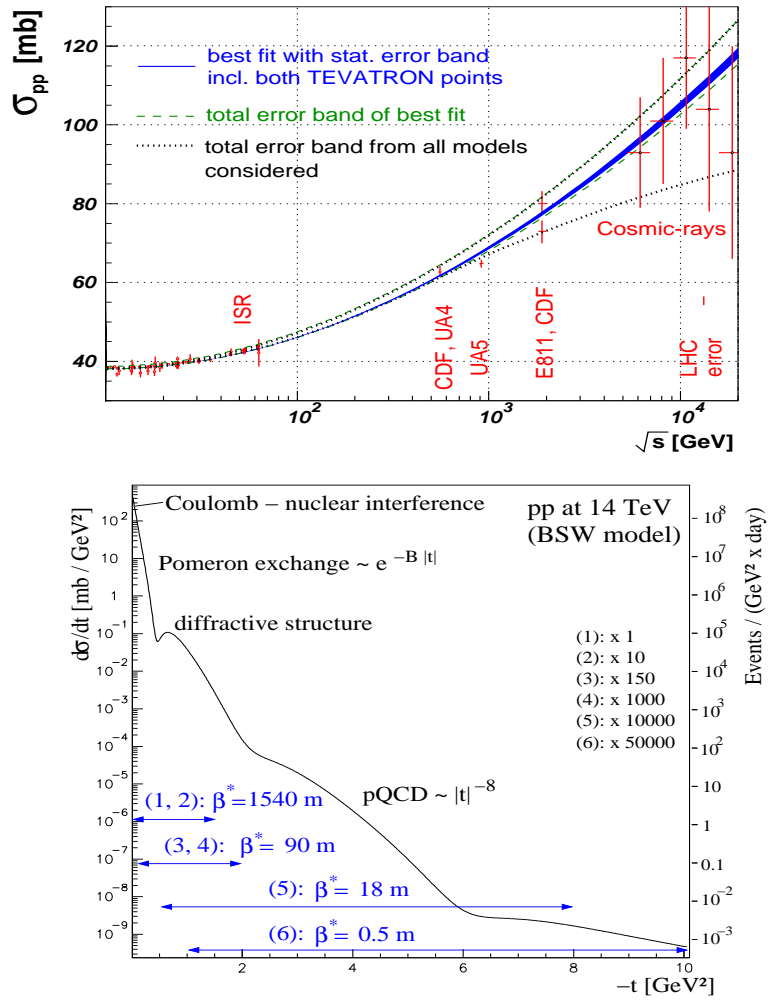

FIG. 5: Top: COMPETE predictions [27] for $\sigma_{t o t}$ with statistical (blue solid) and total (dashed) errors (including the Tevatron ambiguity) compared to existing data. Bottom: Prediction for elastic p-p scattering at the LHC with various beam optics settings [28].

Assuming an uncertainty in $\rho=0.12 \pm 0.02, \sigma_{\text {tot }}$ and $\mathcal{L}$ can be measured within about $1 \%$. Also ATLAS plans to measure the elastic cross section with its Roman Pots [11] based on a fit of the data in the Coulomb region to

$$
\frac{d N}{d t}(t \rightarrow 0)=\mathcal{L} \pi\left(\frac{-2 \alpha}{|t|}+\frac{\sigma_{t o t}}{4 \pi}(i+\rho) e^{-b|t| / 2}\right)^{2} .
$$

Such a measurement requires to go down to $-t \sim 6.5$. $10^{-4} \mathrm{GeV}^{2}$ (i.e. $\theta \sim 3.5 \mu \mathrm{rad}$ ) to reach the kinematical domain where the strong amplitude equals the electromagnetic one.

\subsection{Hard and soft diffractive processes}

Diffractive physics covers the class of inelastic interactions that contain large rapidity gaps (LRGs, $\Delta \eta \gtrsim 4$ ) devoid of hadronic activity and where one or both protons emerge intact in the final state [18]. Such event topologies, with reduced QCD radiation, imply colorless exchange mediated by two or more gluons in a color-singlet state (a Pomeron, $\mathbb{P}$ ). Depending on the number and relative separation of the LRGs, one further differentiates between single, double, or double-Pomeron-exchange (DPE) processes (Fig. 6). The centrally produced system has a mass equal to $M^{2} \approx s \xi_{1}$ $\left(M^{2} \approx s \xi_{1} \xi_{2}\right)$ for single diffractive (DPE) events, and the size 
of the rapidity gap is of the order of $\Delta \eta \sim \log 1 / \xi_{1,2}$.
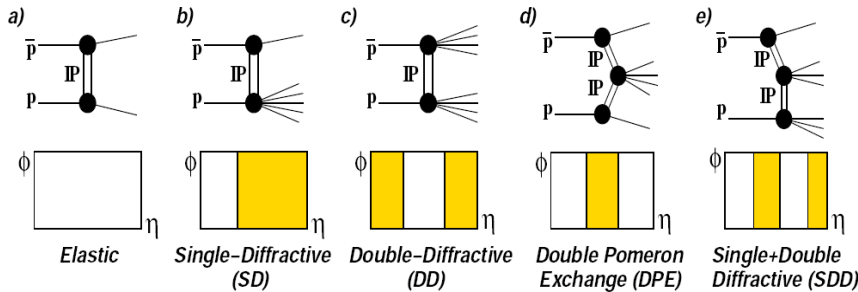

FIG. 6: Event topologies in $\eta$ vs azimuth $\phi$ for elastic and diffractive p-p interactions. Shaded (empty) areas represent particle-production (rapidity-gaps) regions [19].

The interest of diffractive processes is manifold. On the one hand, soft diffraction processes, dominated by nonperturbative (Regge) dynamics, constitute a significant fraction $(\sim 20 \%)$ of the total inelastic $p$ - $p$ cross section. Their characterization - in particular the so-called "rapidity-gap survival probability" - is important in order to have under control the backgrounds of many processes at the LHC [29]. On the other, hard diffraction processes which involve the production of a high-mass or large- $p_{T}$ state $(Q \bar{Q}$, jets, $W, Z \ldots)$ are in principle perturbatively calculable and provide information on diffractive (or generalized) Parton Distribution Functions, dPDFs (GPDs), which describe not only the density of partons in the proton but also their correlations [30].

\subsection{Parton structure and evolution at low- $x$}

Figure 7 summarizes the methods at hand to determine the gluon density $x G\left(x, Q^{2}\right)$ in the proton as a function of fractional momenta $x=p_{\text {parton }} / p_{\text {proton }}$. The main source of information so far on $x G\left(x, Q^{2}\right)$ is (indirectly) obtained from the $\ln Q^{2}$ slope ("scaling violations") of the $F_{2}$ structure function in $e$ $p$ deep-inelastic-scattering (DIS). Additional constraints can be obtained from $F_{2}^{\text {charm }}$ [31] and diffractive photoproduction of heavy vector mesons $(J / \psi, \Upsilon)[32]$ and, in particular (since $x G \propto F_{L}$ ), from the longitudinal structure function $F_{L}$.

For decreasing parton momentum fraction $x$, the gluon density is observed to grow rapidly. As long as the densities are not too high, this growth is described by the Dokshitzer-Gribov-Lipatov-Altarelli-Parisi (DGLAP) [33] or by the Balitski-Fadin-Kuraev-Lipatov (BFKL) [34] evolution equations which govern, respectively, parton radiation in $Q^{2}$ and $x$ (Fig. 8). Eventually, at high enough center-of-mass energies (i.e. at very small $x$ ) the gluon density will be so large that non-linear (gluon-gluon fusion) effects will become important [35]. A regime of saturated parton densities is thus expected for small enough $x$ values at virtualities below an energy-dependent "saturation momentum" scale, $Q_{s}$, intrinsic to the size of the hadron. Saturation effects are amplified in nuclear targets because of their increased transverse parton density compared to the proton (for nuclei, $Q_{s}^{2} \propto A^{1 / 3}$ where $A \approx 200$ is the mass number in $P b$ or $A u$ ) [20].

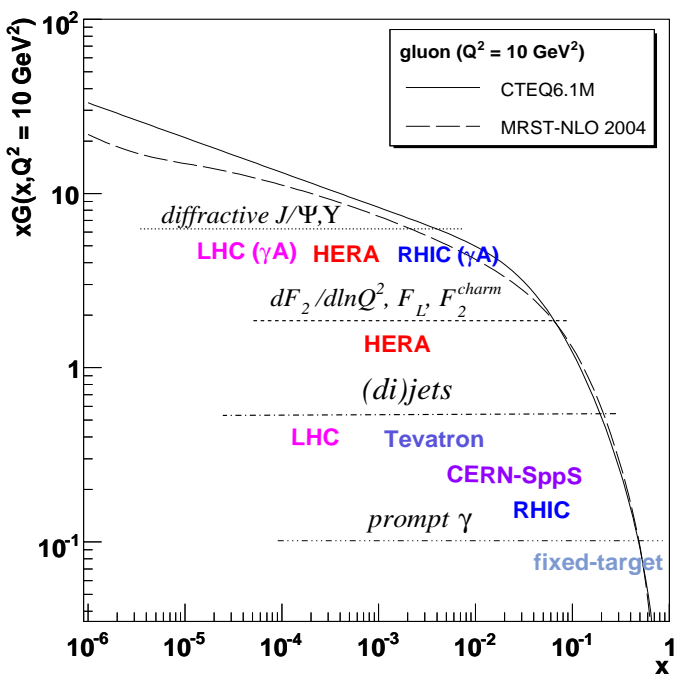

FIG. 7: Examples of measurements providing information on the gluon PDF as a function of $x$ [21].

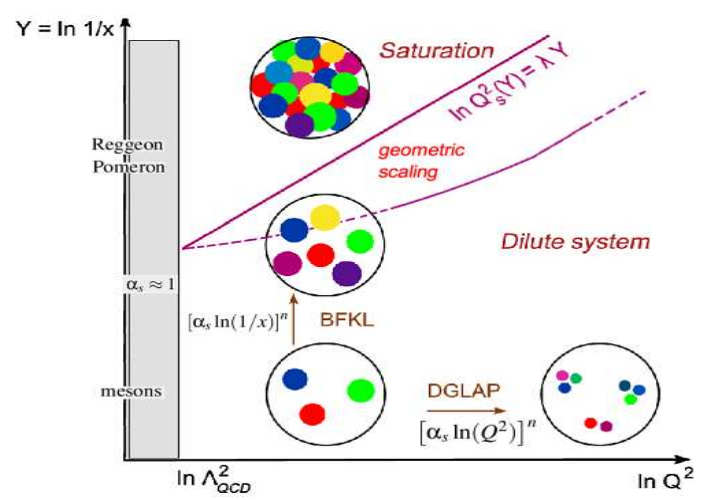

FIG. 8: $\log (1 / x)-\mathrm{Q}^{2}$ plane with the different QCD evolution regimes (DGLAP, BFKL, saturation).

In hadron-hadron collisions, information on $x G$ can be obtained in processes with prompt photons, jets, and heavyquarks in the final state. In a $2 \rightarrow 2$ parton scattering the minimum momentum fraction probed when a particle of momentum $p_{T}$ is produced at pseudo-rapidity $\eta$ is $x_{\text {min }}=$ $2 p_{T} / \sqrt{s} \exp (-\eta)$, i.e. $x_{\min }$ decreases by a factor of $\sim 10$ every 2 units of rapidity. Thus, forward instrumentation provides an important lever arm for the measurement of the low- $x$ structure and evolution of the parton densities. Three representative low- $x$ QCD measurements at the LHC [21] are discussed next.

\section{- Case study I: Forward (di)jets}

From the formula for $x_{\min }$ above, it follows that the measurement of relatively soft jets with $E_{T} \approx 20-100 \mathrm{GeV}$ in the forward calorimeters $(3<|\eta|<6.6)$ allows one to probe the PDFs at $x$ values as low as $10^{-5}$, in $p$ - $p$ at $14 \mathrm{TeV}$. In the low side of this kinematic range, the current PDF uncertain- 
ties result in variations of the jet cross sections as large as $60 \%$ (Fig. 9, top). The interest in forward jet measurements goes beyond the single inclusive cross sections: the production of dijets with similar $E_{T}$ but separated by large rapidities, the so-called "Mueller-Navelet jets" (diagram (a) of Fig. 1) [37], is a particularly sensitive measure of BFKL $[38,39]$ as well as non-linear [40, 41] QCD evolutions. The large rapidity interval between the jets (e.g. up to $\Delta \eta \approx 12$ in the extremes of CMS forward calorimeters) enhances large logarithms of the type $\Delta \eta \sim \log \left(s / E_{T, 1} E_{T, 2}\right)$ which can be appropriately resummed using the BFKL equation. One of the phenomenological implications of BFKL dynamics is an enhanced radiation between the two jets which results in a larger azimuthal decorrelation for increasing $\Delta \eta$ separations, compared to collinear pQCD approaches. Preliminary CMS analyses [36] indicate that such studies are well feasible by measuring jets in each one of the HF forward calorimeters (Fig. 9, bottom).
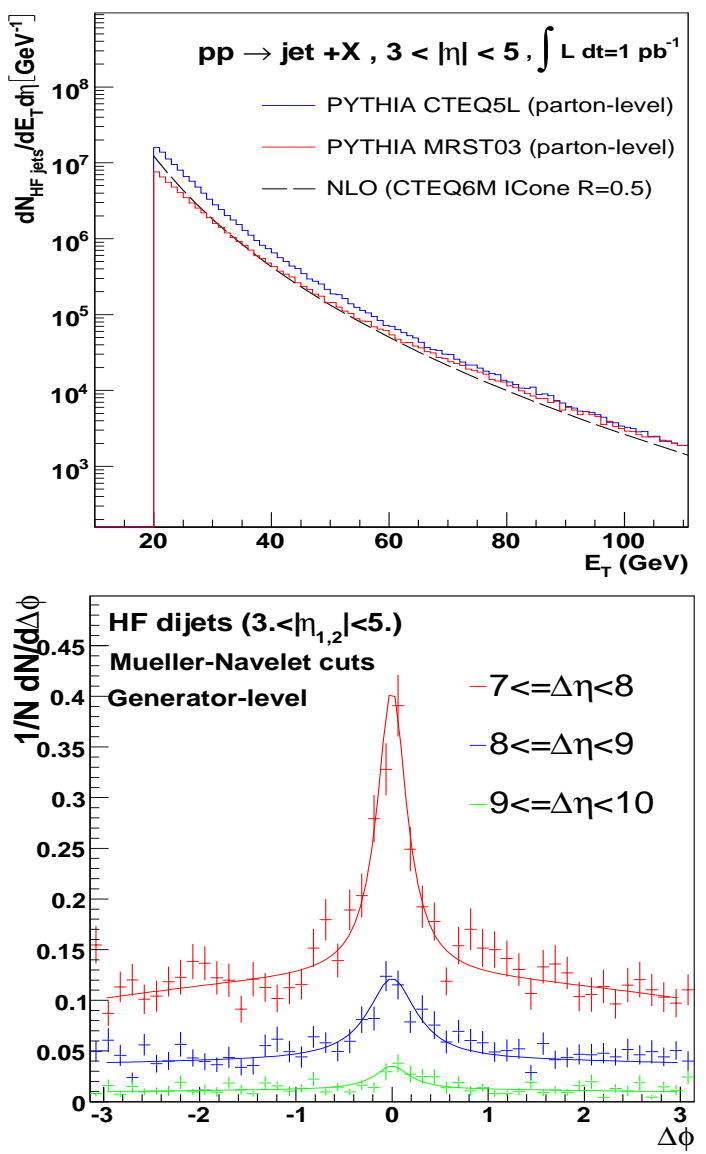

FIG. 9: Forward jet results in $\left(1 \mathrm{pb}^{-1}\right) \quad p-p$ collisions at $\sqrt{s}=14 \mathrm{TeV}$ [36]. Top: Single inclusive jet yields in the CMS forward calorimeters expected with various PDF sets. Bottom: $\Delta \phi=\left(\phi_{1}-\phi_{2}\right)-\pi$ distributions for dijet events passing the MuellerNavelet cuts with separations $\Delta \eta=7.5,8.5$ and 9.5.
- Case study II: Forward heavy-quarks

The possibility of ALICE and LHCb (Fig. 10, top) to reconstruct heavy $D$ and $B$ mesons as well as quarkonia in a large forward rapidity range can also add stringent constraints on the gluon structure and evolution at low- $x$. Studies of small$x$ effects on heavy flavor production based on collinear and $k_{T}$ factorization, including non-linear terms in the parton evolution, lead to varying predictions for the measured $c$ and $b$ cross sections at the LHC [31]. The hadroproduction of $J / \psi$ proceeds mainly via gluon-gluon fusion and, having a mass around the saturation scale $Q_{s} \approx 3 \mathrm{GeV}$ at the LHC, is also a sensitive probe of possible gluon saturation phenomena. Figure 10 (bottom) shows the gluon $x$ range probed in $p$ - $p$ collisions producing a $J / \psi$ inside the ALICE muon arm acceptance $(2.5 \lesssim \eta \lesssim 4)$. The observed differences in the underlying PDF fits translate into variations as large as a factor of $\sim 2$ in the finally measured cross sections [42].
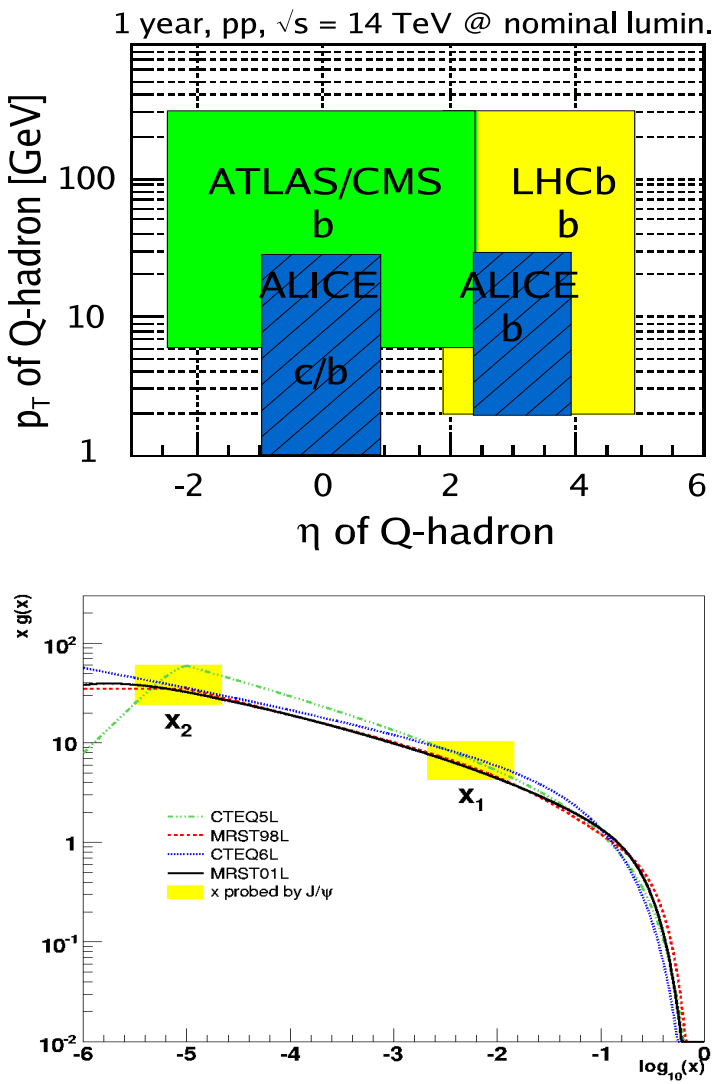

FIG. 10: Top: Acceptances in $\left(\eta, p_{T}\right)$ for open charm and bottom at the LHC [31]. Bottom: Sensitivity of the forward $J / \psi$ measurement in ALICE to various $x$ ranges of the gluon PDF [42].

\section{- Case study III: $Q \bar{Q}$ exclusive photoproduction}

High-energy photons from electromagnetic (ultraperipheral) proton-proton or ion-ion interactions can be used to constrain the low- $x$ behavior of the nuclear gluon density via ex- 
clusive photoproduction of quarkonia, dijets and other hard processes [23]. This is particularly interesting with heavyions where, thanks to the large nuclear charge $(Z=80$ for $\mathrm{Pb})$, the available photon fluxes $\left(d N_{\gamma} / d \omega \propto Z^{2}\right)$ allow one to precisely probe the barely known nuclear gluon distribution (Fig. 11, top). Lead beams at $2.75 \mathrm{TeV}$ have Lorentz factors $\gamma=2930$ leading to maximum (equivalent) photon energies $\omega_{\max } \approx \gamma / R \sim 100 \mathrm{GeV}$, and corresponding maximum c.m. energies: $W_{\gamma \gamma}^{\max } \approx 160 \mathrm{GeV}$ and $W_{\gamma-\mathrm{A}}^{\max } \approx 1 \mathrm{TeV}$, i.e. 3-4 times higher than equivalent photoproduction studies at HERA. The $x$ values probed in $\gamma$-A $\rightarrow Q \bar{Q} A$ processes (diagram (b) of Fig. 1) can be as low as $x \sim 10^{-5}$ [43]. ALICE, ATLAS and CMS can measure the $J / \psi, \Upsilon \rightarrow e^{+} e^{-}, \mu^{+} \mu^{-}$produced in electromagnetic $P b-P b$ collisions tagged with neutrons detected in the ZDCs. Full simulation analyses [44] indicate that CMS can measure a total yield of $\sim 500 \Upsilon$ 's within $|\eta|<2.5$ for the nominal $0.5 \mathrm{nb}^{-1} \mathrm{~Pb}-\mathrm{Pb}$ integrated luminosity (Fig. 11, bottom). With such statistics, studies of the $p_{T}$ and $\eta$ distributions of the $\Upsilon$ can be carried out which will help constrain the low- $x$ gluon density in the $P b$ nucleus. Similar exclusive $\Upsilon$ studies have been conducted in $p-p$ collisions [45].
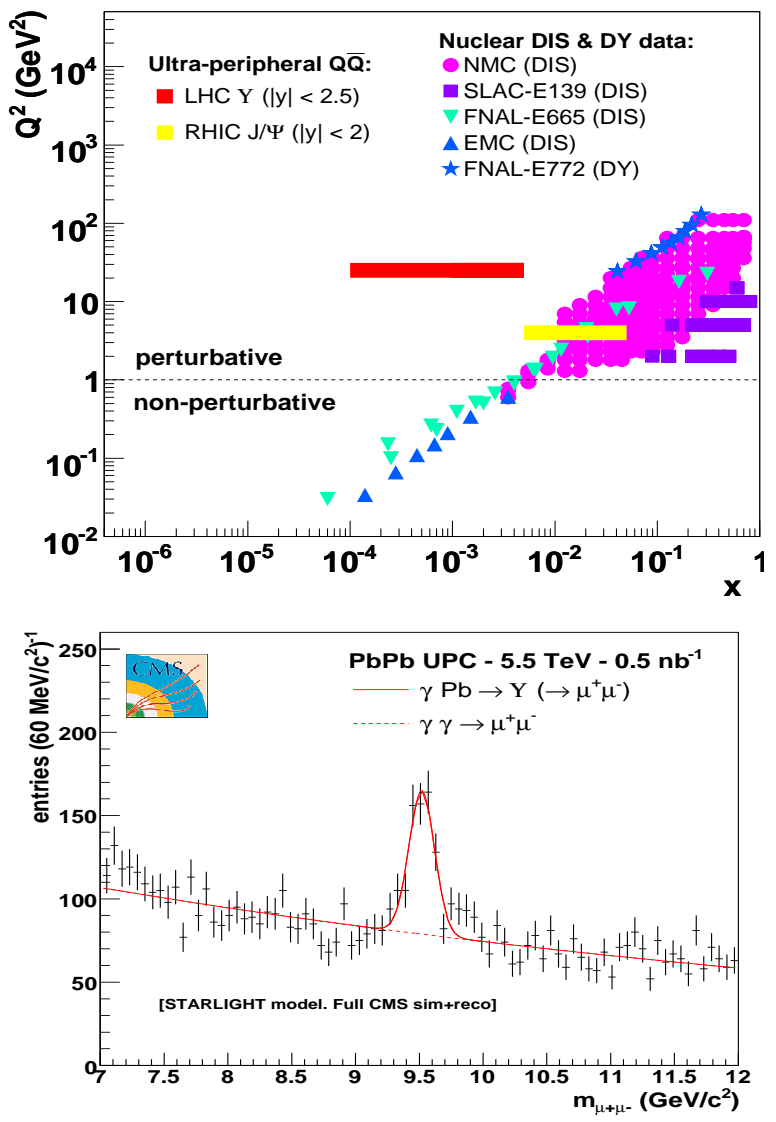

FIG. 11: Top: Kinematic $\left(x, Q^{2}\right)$ plane probed in e- $\gamma$-A collisions: DIS data compared to ultraperipheral $Q \bar{Q}$ photoproduction ranges [43]. Bottom: Expected dimuon invariant mass from $\gamma P b \rightarrow$ $\Upsilon P b^{\star}$ on top of the $\gamma \gamma \rightarrow \mu^{+} \mu^{-}$continuum in ultraperipheral $P b-P b$ collisions at $\sqrt{s_{N N}}=5.5 \mathrm{TeV}$ [44].
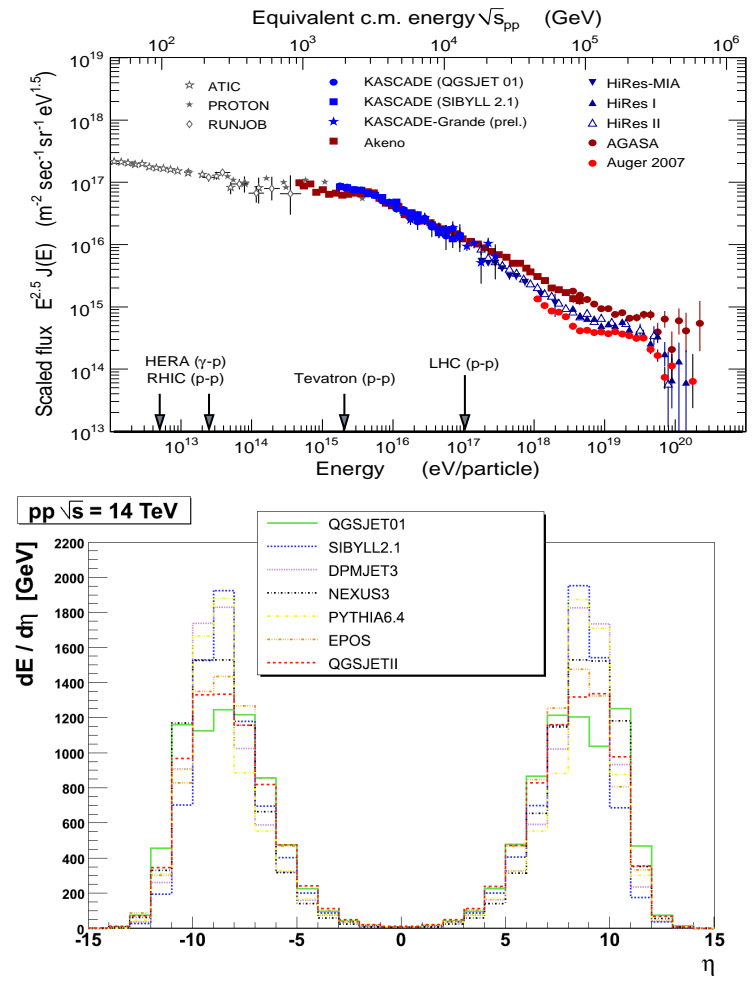

FIG. 12: Top: Measured CR energy spectrum. Bottom: Pseudorapidity energy density for $p$ - $p$ at $\sqrt{s}=14 \mathrm{TeV}$ predicted by various commonly used MC models in UHE cosmic rays physics [24].

\subsection{Validation of QCD Monte Carlos for UHE cosmic-rays}

The LHC will not only address fundamental open questions in particle physics but will also provide valuable insights in closely related domains, such as on the origin and nature of cosmic rays (CRs) with energies between $10^{15} \mathrm{eV}$ and the so-called "GZK-cutoff" at $10^{20} \mathrm{eV}$, recently measured by the HiRes [46] and Auger [47] experiments (Fig. 12, top). CR candidates are protons and nuclei as massive as iron, which generate "extended air-showers" in proton-nucleus ( $p$-Air) and nucleus-nucleus ( $\alpha$-,Fe- Air) collisions when entering the atmosphere. Determination of the primary energy and mass relies on hadronic Monte Carlo codes which describe the interactions of the primary (dominated by forward and soft QCD interactions) in the upper atmosphere [24]. Existing MC models predict energy and multiplicity flows differing by factors as large as three, with significant inconsistencies in the forward region. The measurement of forward particle production in $p$ - $p, p-A$ and $A-A$ collisions ${ }^{2}$ at LHC energies (equivalent to $E_{\text {lab }} \approx 10^{17} \mathrm{eV}$ ) will provide strong constraints on these models and allow for more reliable extrapolations of the CR energy and composition around the GZK cut-off. Figure 12 (bottom) compares the predictions of QGSJET [48], DPM-

\footnotetext{
2 Note that CRs interactions in the atmosphere are mostly proton-nucleus ( $p$-Air) and nucleus-nucleus ( $\alpha$-,Fe- Air) collisions.
} 
JET [1], NEXUS [49], EPOS [50], and PYTHIA [51] for the energy flow $(d E / d \eta)$ in $p-p$ collisions at $\sqrt{s}=14 \mathrm{TeV}$. In the range covered by detectors like CASTOR or TOTEM (around $|\eta| \approx 6$ ) and ZDC or LHCf (beyond $|\eta| \approx 8$, for neutrals), the model predictions differ by up to $\sim 60 \%$.

\section{ELECTROWEAK PHYSICS}

\subsection{Beam luminosity via exclusive dileptons}

Two-photon dilepton production, $p p \rightarrow p l^{+} l^{-} p$ (diagram (c) of Fig. 1) is a useful luminosity calibration process, thanks to its precisely known (nearly pure) QED cross section [52]. Experimentally, such a process can be tagged with rapidity-gaps ("exclusivity") conditions [45] or via forward protons [22], and has a clear signature in the exclusive back-to-back dileptons $\left(\left|\Delta \phi\left(l^{+} l^{-}\right)\right|>2.9\right)$ measured within $|\eta|<3$. The LPAIR [53] cross section for events where both muons have $p_{T}>3 \mathrm{GeV} / \mathrm{c}$ and can, therefore, reach the CMS muon chambers is about $50 \mathrm{pb}$. About 710 exclusive dimuons per $100 \mathrm{pb}^{-1}$ are expected in CMS after selection cuts [45]. The resulting invariant mass distribution is shown in Fig. 13, together with the background muons from the $\Upsilon$ decay and inelastic (or dissociative) dimuon events. This measurement is also easily accessible in electromagnetic $\mathrm{Pb}-\mathrm{Pb}$ collisions (see Fig. 11 bottom) where the dilepton continuum (thanks to the $Z^{4}$ photon flux enhancement factor) is much larger than in $p-p$.

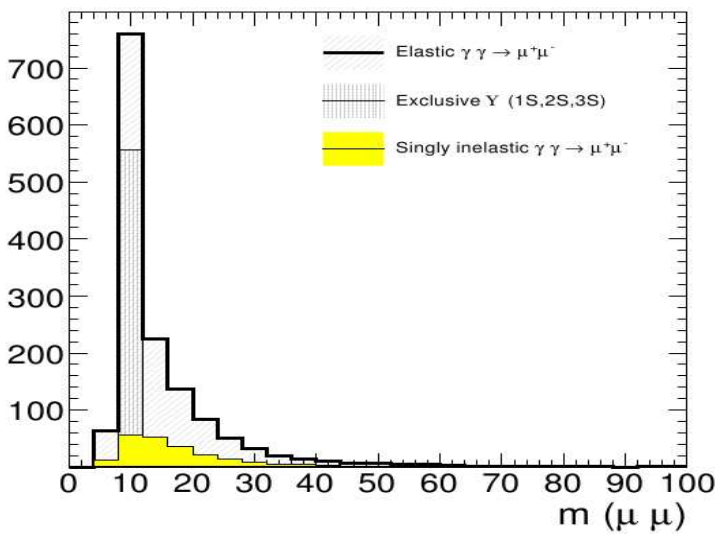

FIG. 13: Dimuon mass distribution in photon-induced events (for $100^{-1} \mathrm{pb}$ in $p-p$ at $\sqrt{s}=14 \mathrm{TeV}$ ) passing the exclusivity cuts discussed in [45].

\subsection{New physics via anomalous gauge boson couplings}

The study of the couplings of the gauge bosons $(\gamma, W$ and $Z$ ) among themselves provides the most direct (yet difficult) way to test the gauge structure of the electroweak theory. Many physics scenarios beyond the SM, with novel interactions and/or particles, lead to modifications of the gauge boson self-interaction vertices. A process well-suited to testing the triple $W W \gamma$ vertex is the photoproduction of single $W$ bosons from a nucleon in ultra-peripheral $p-p$ [22] and $A-A$ [54] collisions tagged with forward protons or neutrons (Fig. 14). A large cross section of about $1 \mathrm{pb}$ is expected for large photonproton c.m. energies, $W_{\gamma p}>1 \mathrm{TeV}$. In addition, the exclusive two-photon production of $W^{+} W^{-}$pairs probes quartic gaugeboson-couplings. The process has a total cross section of more than $100 \mathrm{fb}$, and a very clear signature. Its cross section is still about $10 \mathrm{fb}$ for $W_{\gamma p}>1 \mathrm{TeV}$ showing sensitivity to physics beyond the SM [22].

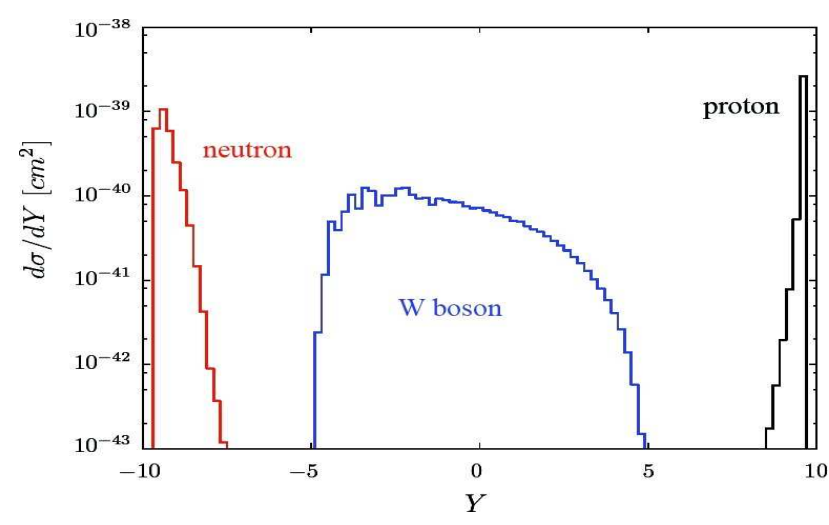

FIG. 14: Rapidity distribution of single- $W$ photoproduction [54] in $p$ - $p$ at $\sqrt{s}=14 \mathrm{TeV}$ - note that the forward protons (neutrons) fall within the RP (ZDC) acceptances.

\section{HIGGS PHYSICS}

\subsection{Vector Boson Fusion production}

The second most important production channel of the SM Higgs boson at the LHC (Fig. 15), is the vector-boson-fusion (VBF) process where two $W$ or $Z$, radiated off valence quarks, merge to produce a Higgs. The distinctive signature of VBF is the presence of two forward-backward jets from the fragmentation of the two incoming quarks. The importance of forward calorimetry proves crucial to select VBF events. The average separation of these jets is $\Delta \eta \approx 5$ and, thus, VBF events are usually tagged in ATLAS and CMS by jets detected in the FCal and HF calorimeters. Such a distinct event topology reduces efficiently a significant fraction of the Higgs backgrounds (e.g. $t \bar{t}, W W$ or $W, Z+n j$ which are characterized by jets at central rapidities, see Fig. 16) making the $q q H \rightarrow q q W W$ and $q q H \rightarrow q q \tau \tau$ discovery channels at the LHC $[55,58]$.

\subsection{Central exclusive production}

Central exclusive production (CEP) is defined as a process of the type $p p \rightarrow p \oplus X \oplus p$, where $X$ is a fully measured simple state such as $\chi_{c, b}$, jet-jet $(j j), \gamma \gamma, \mathrm{H}, \ldots$ and ' $\oplus$ ' represents a large rapidity-gap $(\Delta \eta \gtrsim 4)$. Central exclusive Higgs 


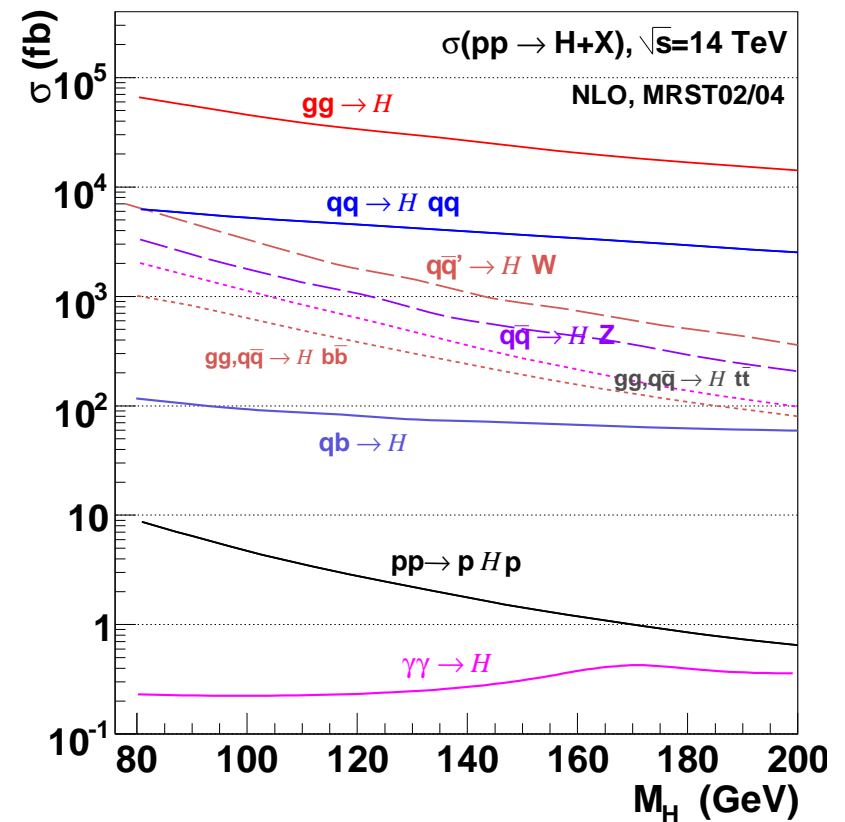

FIG. 15: Cross sections for the SM Higgs versus $M_{h}$ in nine different production channels at the LHC, from [56] $\left(\sigma_{p H p}\right.$ is from [12], and $\sigma_{\gamma \gamma \rightarrow H}$ from [57]).

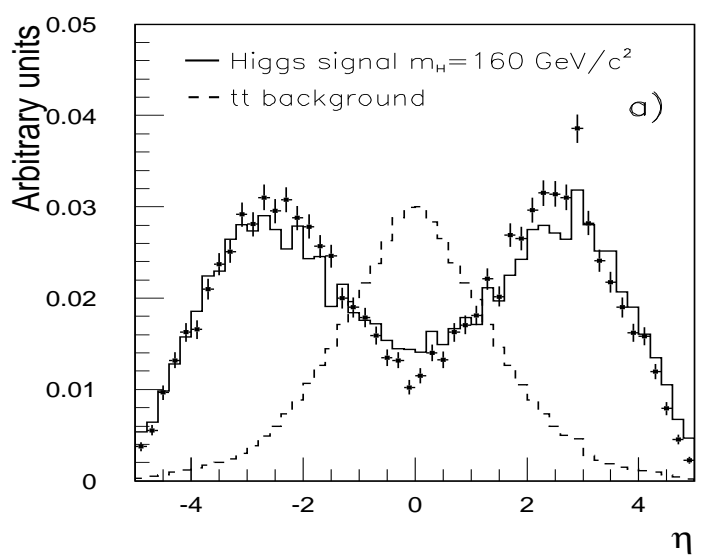

FIG. 16: Typical pseudorapidity distribution of the jets in VBF Higgs events and in $t \bar{t}$ background events [58].

production (diagram (d) of Fig. 1) has attracted significant experimental and theoretical interest for various important reasons $[12,25]$. First, to a very good approximation in CEP the primary active di-gluon system obeys a $J_{z}=0$, $\mathrm{C}$-even, P-even, selection rule (where $J_{z}$ is the projection of the total angular momentum along the proton beam axis). This selection rule readily permits a clean determination of the quantum numbers of any new resonance by measuring the azimuthal correlations of the scattered protons. Second, because the process is exclusive, the energy loss of the outgoing protons is directly related to the invariant mass of the central system, $M^{2} \approx s \xi_{1} \xi_{2}$, allowing an excellent mass measurement $\left(\sigma_{M} \sim 2 \mathrm{GeV} / \mathrm{c}^{2}\right)$ irrespective of the decay mode of the central system. Third, thanks to the spin selection rule a large fraction of QCD production is suppressed resulting in a very favorable 1:1 signal-to-background. The expected SM cross sections are of order 3-10 fb (Fig. 15) although, in certain regions of the minimal supersymmetric extension of the SM (MSSM, at high $\tan \beta$ and small $M_{A}$ ) with enhanced Higgs coupling to fermions, they can be a factor of 10-100 larger [59].

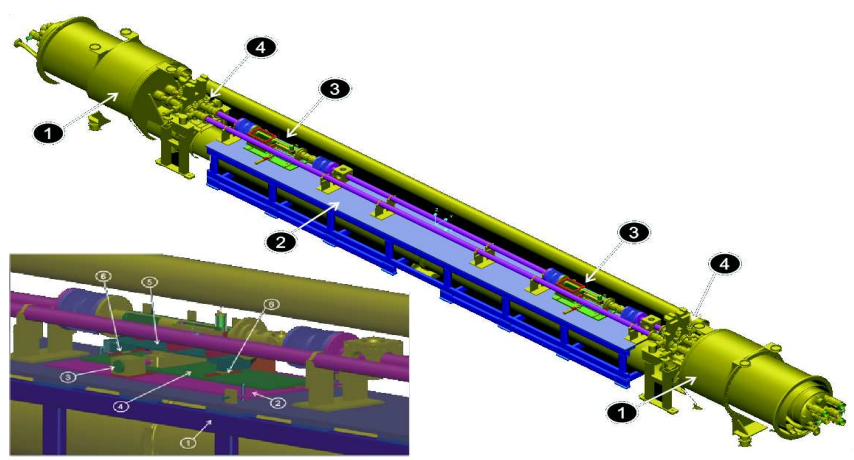

FIG. 17: Top view of the proposed FP420 system at $420 \mathrm{~m}$ from ATLAS and CMS IPs (zoom shows the support table with one detector section) [12].

For a Higgs mass close to the LEP limit, $M_{H} \approx 120 \mathrm{GeV} / \mathrm{c}^{2}$, the optimal proton tagging acceptance is beyond the current Roman Pots at 220,240 m. The proposed FP420 detector system [12] - a magnetic spectrometer consisting of a moveable silicon tracking system and fast Čerenkov detectors located in a 12-m region at about $420 \mathrm{~m}$ from the ATLAS and CMS IPs (Fig. 17) - allows for the detection of both outgoing protons scattered by a few hundreds $\mu$ rads (i.e. $3-9 \mathrm{~mm}$ ) relative to the LHC beam line. A measurement of the protons relative time of arrival in the $10 \mathrm{ps}$ range is required for matching them with a central vertex within $\sim 2 \mathrm{~mm}$, which will enable the rejection of a large fraction of the pile-up background at high-luminosities. Under such circumstances, the Higgs boson line-shape can be reconstructed in the otherwise (difficult) $b \bar{b}$ channel with a $3 \sigma$ or better significance with an integrated luminosity of $60 \mathrm{fb}^{-1}$ (Fig. 18).

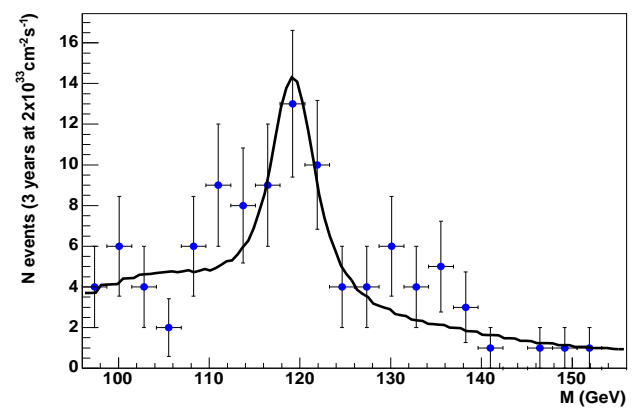

FIG. 18: Expected mass fit for the MSSM $h \rightarrow b \bar{b}$ decay $\left(M_{h}=120 \mathrm{GeV} / \mathrm{c}^{2}\right)$, measured with FP420 in $60 \mathrm{fb}^{-1}$ integrated luminosity $[12,60]$. (The significance of the fit is $3.5 \sigma$ ). 


\section{Summary}

Many interesting scattering processes at the LHC - mediated by colorless exchanges (photons, heavy gauge-bosons, Pomerons, di-gluons in a color-single-state) - are open to study at $\mathrm{TeV}$ energies for the first time, thanks to detector instrumentation at low angles with respect to the beam. We have reviewed the near-beam instrumentation capabilities (at pseudo-rapidities $|\eta|>3$ ) and the associated "forward" physics programme of the various LHC experiments in various sectors and extensions of the Standard Model. The varied and unique set of measurements accessible in the QCD (diffractive, low- $x$, hadronic MCs for cosmic-rays), electroweak (exclusive dileptons, gauge couplings), Higgs (vector-boson-fusion and exclusive production) and beyond the SM (anomalous couplings, exclusive MSSM Higgs) sectors, provides a powerful and complementary way to explore the particles and interactions of nature at energies never reached before.

\section{Acknowledgments}

I would like to thank the organisers of LAWHEP'07 - in particular Beatriz Gay-Ducati, Magno Machado and Carlos A. Garcia-Canal - for their kind invitation to an stimulating physics school. Special thanks due to Michele Arneodo and Albert de Roeck for valuable discussions. Work supported by the 6th EU Framework Programme contract MEIF-CT-2005025073 .
[1] S. Roesler, R. Engel and J. Ranft, arXiv:hep-ph/0012252 (2000).

[2] R. Orava, J. Lamsa, Diffraction'06 Int. Workshop (2006).

[3] ATLAS: Detector and Physics performance TDR, CERN/LHCC-99-14 (1999).

[4] CMS Physics TDR, Volume I: Detector Performance and Software, CERN/LHCC-2006-001(2006).

[5] M. Albrow et al. [CMS/TOTEM Collabs.], CERN-LHCC2006-039 (2006)

[6] A. S. Ayan et al., J. Phys. G 30 N33 (2004).

[7] X. Aslanoglou et al., Eur. Phys. J. C 52, 495 (2007).

[8] S.N. White et al. [ATLAS Collaboration], CERN-LHCC-2007001.

[9] O. A. Grachov et al. [CMS Collaboration], AIP Conf. Proc. 867, 258 (2006).

[10] C. Royon [RP220 Collaboration], arXiv:0706.1796 [physics.ins-det].

[11] S. Ask [ATLAS Collaboration], Proceeds. DIS'07 arXiv:0706.0644 [hep-ex].

[12] M.G. Albrow et al. [FP420 R\&D Collaboration], arXiv:0806.0302 [hep-ex].

[13] F. Carminati et al. [ALICE Collaboration], J. Phys. G 30, 1517 (2004).

[14] LHCb, Reoptimised Detector Design and Performance TDR, CERN/LHCC 2003-030, (2003).

[15] ALICE TDR: Zero degree calorimeter (ZDC), CERN-LHCC99-05 (1999).

[16] V. Berardi et al. [TOTEM Collaboration], CERN/LHCC-2004002 (2004).

[17] Technical Design Report of the LHCf experiment, CERNLHCC-2006-004 (2006).

[18] M. Arneodo and M. Diehl, arXiv:hep-ph/0511047 (2005).

[19] K. Goulianos, arXiv:0707.1055 [hep-ex] (2007).

[20] See e.g. F. Gelis, T. Lappi and R. Venugopalan, Int. J. Mod. Phys. E 16, 2595 (2007).

[21] D. d'Enterria, Eur. Phys. J. A 31, 816 (2007).

[22] J. Favereau et al., CP3-08-04 (June 2008), to be submitted to Eur. Phys. J. C.

[23] A. Baltz et al., Phys. Rept. 458, 1 (2008).

[24] D. d'Enterria, R. Engel. T. McCauley and Tanguy Pierog, Proceeds. QM08; arxiv:0806.0944.

[25] A. De Roeck, V. A. Khoze, A. D. Martin, R. Orava, and M. G. Ryskin, Eur. Phys. J. C 25, 391 (2002).
[26] C. Bourrely, N. N. Khuri, A. Martin, J. Soffer and T. T. Wu, arXiv:hep-ph/0511135 (2005).

[27] J. R. Cudell et al. [COMPETE Collaboration], Phys. Rev. Lett. 89, 201801 (2002).

[28] G. Anelli et al. [TOTEM Collaboration], arXiv:hep-ex/0602025 (2006).

[29] M. G. Ryskin, A. D. Martin and V. A. Khoze, Eur. Phys. J. C 54, 199 (2008).

[30] M. Diehl, Phys. Rept. 388, 41 (2003).

[31] J. Baines et al., Proceeds. HERA-LHC Workshop, hep$\mathrm{ph} / 0601164$ (2006).

[32] A. D. Martin, C. Nockles, M. G. Ryskin and T. Teubner, Phys. Lett. B 662, 252 (2008).

[33] V.N. Gribov and L.N. Lipatov, Sov. Journ. Nucl. Phys. 15, 438 (1972); G. Altarelli and G. Parisi, Nucl. Phys. B 126, 298 (1977); Yu. L. Dokshitzer, Sov. Phys. JETP 46, 641 (1977).

[34] L.N. Lipatov, Sov. J. Nucl. Phys. 23, 338 (1976); E.A. Kuraev, L.N. Lipatov and V.S. Fadin, Zh. Eksp. Teor. Fiz 72, 3 (1977); I.I. Balitsky, L.N. Lipatov, Sov. J. Nucl. Phys. 28, 822 (1978).

[35] L. Gribov, E. M. Levin and M. G. Ryskin, Phys. Rept. 100, 1 (1983); A. H. Mueller and J. w. Qiu, Nucl. Phys. B 268, 427 (1986).

[36] S.Cerci and D. d'Enterria, Proceeds. QM'08, arxiv:0806.0091, Indian J. Phys, to appear.

[37] A. H. Mueller and H. Navelet, Nucl. Phys. B 282, 727 (1987)

[38] V. Del Duca and C. R. Schmidt, Phys. Rev. D 49, 4510 (1994).

[39] A. Sabio Vera, F. Schwennsen, Nucl. Phys. B 776, 170 (2007); Nucl. Phys. B 746, 1 (2006).

[40] C. Marquet and C. Royon, Nucl. Phys. B 739, 131 (2006); and arXiv:0704.3409 [hep-ph].

[41] E. Iancu, M. S. Kugeratski and D. N. Triantafyllopoulos, arXiv:0802.0343 [hep-ph].

[42] D. Stocco [ALICE Collaboration], High energy QCD Workshop, ECT*, Trento (2007).

[43] D. d'Enterria, Proceeds. Photon'07, Nucl. Phys. B Proc. Suppl. to appear; arXiv:0711.1123.

[44] D. d'Enterria (ed.), [CMS Collaboration], J. Phys. G 34, 2307 (2007).

[45] J. Hollar, S. Ovyn and X. Rouby [CMS Collaboration], CMS PAS-DIF-07-001.

[46] R. Abbasi et al. [HiRes Collaboration], Phys. Rev. Lett. 100, 101101 (2008).

[47] T. Yamamoto [Pierre Auger Collaboration], arXiv:0707.2638 
[astro-ph].

[48] S. Ostapchenko, AIP Conf. Proc. 928, 118 (2007), and refs. therein.

[49] T. Pierog et al., Nucl. Phys. A 715, 895 (2003).

[50] K. Werner, T. Pierog, AIP Conf.Proc. 928, 111 (2007), and refs. therein.

[51] T. Sjostrand et al., JHEP 05026 (2006)

[52] D. Bocian and K. Piotrzkowski, Acta Phys. Polon. B 352417 (2004).

[53] J. Vermaseren, Nucl. Phys. B 229, 347 (1983)

[54] U. Dreyer, PhD thesis, Univ. Basel, 2007.
[55] G. L. Bayatian et al. [CMS Collaboration], J. Phys. G 34, 995 (2007).

[56] M. Spira, A. Djouadi, D. Graudenz, and P. M. Zerwas, Nucl. Phys.B 453 (1995).

[57] E. Papageorgiu, Phys. Lett. B 352, 394 (1995).

[58] S. Asai et al. [ATLAS Collaboration], Eur. Phys. J. C 32S2, 19 (2004).

[59] S. Heinemeyer et al., Eur. Phys. J. C 53, 231 (2008).

[60] B. E. Cox, F. K. Loebinger, and A. D. Pilkington, JHEP 0710, 090 (2007). 